\title{
Identification of Material Properties using Full-Field and Non Contact Measurements
}

Universidade Federal Fluminense - UFF Dept. of Mechanical Engineering (PGMEC/LMTA) 24210-240 Niterói, RJ, Brazil

\section{Daniel A. Castello} Member, ABCM

castello@mecanica.coppe.ufrj.br Universidade Federal do Rio de Janeiro - UFRJ

Dept. of Mechanical Engineering 21945-970 Rio de Janeiro, RJ, Brazil

\section{Paulo A. M. dos Santos} pams@if.uff.br Universidade Federal Fluminense - UFF Dept. of Physics 24340-011 Niterói, RJ, Brazil

\section{Carlos F. T. Matt} cfmatt@cepel.br Centro de Pesq. de Energia Elétrica - CEPEL Dept. of Lines and Substations 21941-590 Rio de Janeiro, RJ, Brazil
In the present work, the Digital Image Correlation (DIC) measurement method is used to obtain the displacement field of specific regions of a cantilever beam under bending. These fields are used within an inverse analysis scheme in order to obtain the elasticity modulus of the beam material. The parameter estimation is performed by means of the minimization of an error function comprising of the difference between the displacement fields obtained from the experiment and from an appropriate mathematical model. The inverse problem is solved by means of the classical Levenberg-Marquardt nonlinear parameter estimation technique. The estimate obtained for the elasticity modulus is validated taking into account new experimental data obtained through modal analysis of another beam-like specimen which, in turn, is made of the same material as the original one.

Keywords: full-field measurement, digital image correlation, inverse problem, parameter estimation

\section{Introduction}

The use of inverse problems in experimental mechanics has had an enormous increase in the last decade and its applications embrace different areas, such as heat transfer (Özişik and Orlande, 2000), structural dynamics (Stutz et al., 2005), acoustics (Leiderman et al., 2007), to name just a few. There has always been a demand to seamlessly integrate experimental information with analytical or numerical models. Efforts for this integration have gained an important ally with the advent of experimental methods that are capable of generating full-field measurements.

Optical methods, such as shearography, laser speckle interferometry and moiré can be employed to measure full-field surface displacement and strain (Burke et al., 2007; Pandurangan and Buckner, 2007; Cardenas-Garcia and Preidikman, 2006). All of these methods have been combined with image processing and electronic systems and developed with commercial scientific instruments. However, some types of equipment based on these optical methods are still very expensive and require stable environment as well as laborious data reduction processes. On the other hand, the digital image correlation (DIC) measurement method (McGinnis et al., 2005; Corr et al., 2007; Wang et al., 2002; Lecompte et al., 2007) represents a different approach that is less demanding optically: incoherent light is sufficient; a vibration isolation table is not required and the optical components are eliminated. Recently, the use of DIC method to measure full-field surface displacement has increased considerably due to the development of high resolution Charge Coupled Device (CCD) cameras.

The aim of the present work is to estimate the elasticity modulus of a cantilever beam-like specimen through an inverse analysis based on full-field displacement measurements obtained out of a simple experimental set-up. The inverse analysis scheme combines the beam full-field displacement captured by the digital image correlation method and the Levenberg-Marquardt parameter estimation method to solve the associated inverse problem. The estimate obtained for the elasticity modulus is validated taking into account new experimental data obtained through modal analysis of another beam-like specimen, which, in turn, is made of the same material as the original one.

\begin{tabular}{|c|c|}
\hline \multicolumn{2}{|c|}{ Nomenclature } \\
\hline$b$ & $=$ beam width, $\mathrm{m}$ \\
\hline$f_{j}$ & $=j$-th estimated natural frequency, $\mathrm{Hz}$ \\
\hline$f_{j}^{E x p}$ & $=j$-th measured natural frequency, $\mathrm{Hz}$ \\
\hline$f(x, y)$ & $=$ pixel gray level at coordinates $(x, y)$ \\
\hline$g\left(x^{\prime}, y^{\prime}\right)$ & $=$ pixel gray level at coordinates $\left(x^{\prime}, y^{\prime}\right)$ \\
\hline$h$ & $=$ beam height, $\mathrm{m}$ \\
\hline $\mathbf{p}$ & $=$ vector of unknown parameters \\
\hline$u$ & $=$ displacement field along $x$-direction, $\mathrm{m}$ \\
\hline$v$ & $=$ displacement field along $y$-direction, $\mathrm{m}$ \\
\hline$x_{s}$ & $=x$-coordinate of starting point, $\mathrm{m}$ \\
\hline $\mathbf{y}^{E}(\mathbf{x}, t)$ & $=$ experimental response \\
\hline $\mathbf{y}(\mathbf{p} ; \mathbf{x}, t)$ & $=$ estimated response \\
\hline$C(u, v)$ & $=$ correlation coefficient \\
\hline$E$ & $=$ elasticity modulus, $\mathrm{Pa}$ \\
\hline$F$ & $=$ applied force, $\mathrm{N}$ \\
\hline$I$ & $=$ beam cross-section moment of inertia, $\mathrm{m}^{4}$ \\
\hline $\mathbf{J}$ & $=$ sensitivity matrix \\
\hline$L$ & $=$ beam length, $\mathrm{m}$ \\
\hline$N_{p}$ & $=$ number of unknown parameters \\
\hline$N_{s}^{p}$ & $=$ number of sensors \\
\hline$N_{t}$ & $=$ number of measured data \\
\hline$S$ & $=$ ordinary least squares norm \\
\hline $\mathbf{Y}$ & $=$ vector containing the estimated response \\
\hline$\overline{\mathbf{Y}}$ & $=$ vector containing the measured response \\
\hline
\end{tabular}




\section{Greek Symbols}

$$
\begin{array}{ll}
\varepsilon_{1}, \varepsilon_{2}, \varepsilon_{3} & =\text { user-prescribed tolerances } \\
\mu & =\text { numerical damping parameter } \\
\rho & =\text { beam mass per unit length, } \mathrm{kg} / \mathrm{m} \\
\Delta f & =\text { relative error between measured and } \\
\Gamma & \text { estimated natural frequencies } \\
& =\text { Levenberg-Marquardt's damping matrix }
\end{array}
$$

\section{Subscripts/Superscripts}

$\begin{array}{ll}k & \text { relative to iteration number } \\ s & \text { relative to sensor number } \\ E, \operatorname{Exp} & \text { relative to experiment }\end{array}$

\section{Digital Image Correlation (DIC) Method}

The Digital Image Correlation (DIC) is an optical-numerical full-field surface displacement measurement method. It is based on a comparison between two images of the specimen coated by a random speckle pattern in the undeformed and in the deformed state. Its special merits encompass non-contact measurement, simple optic setup, no special preparation of specimens and no special illumination.

The basic principle of the DIC method is to search for the maximum correlation between small zones (subwindows) of the specimen in the undeformed and deformed states, as illustrated in Fig. 1. From a given image-matching rule, the displacement field at different positions in the analysis region can be computed. The simplest image-matching procedure is the cross-correlation, which provides the in-plane displacement fields $u(x, y)$ and $v(x, y)$ by matching different zones of the two images.

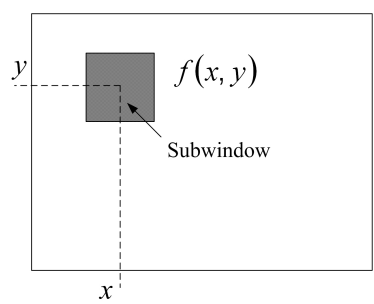

Underformed state

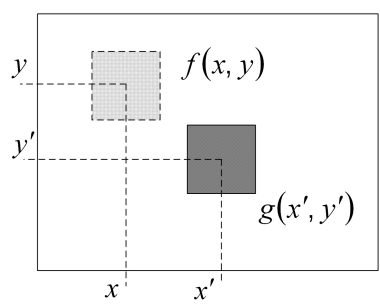

Derformed state
Figure 1. Schematic diagram of the deformation relation.

A commonly used correlation coefficient is defined as follows (Dally and Riley, 2005):

$$
C(u, v)=\frac{\sum_{i=1}^{m} \sum_{j=1}^{m}\left[f\left(x_{i}, y_{j}\right)-\bar{f}\right]\left[g\left(x_{i}^{\prime}, y_{j}^{\prime}\right)-\bar{g}\right]}{\sqrt{\sum_{i=1}^{m} \sum_{j=1}^{m}\left[f\left(x_{i}, y_{j}\right)-\bar{f}\right]^{2}} \sqrt{\sum_{i=1}^{m} \sum_{j=1}^{m}\left[g\left(x_{i}^{\prime}, y_{j}^{\prime}\right)-\bar{g}\right]^{2}}}
$$

where

$$
\begin{aligned}
& x^{\prime}=x+u_{0}+\frac{\partial u}{\partial x} d x+\frac{\partial u}{\partial y} d y \\
& y^{\prime}=y+v_{0}+\frac{\partial v}{\partial x} d x+\frac{\partial v}{\partial y} d y
\end{aligned}
$$

$f(x, y)$ is the pixel gray level value (ranging from 0 to 255) at the coordinates $(x, y)$ for the undeformed or original image; $g\left(x^{\prime}, y^{\prime}\right)$ is the pixel gray level value at the coordinates $\left(x^{\prime}, y^{\prime}\right)$ for deformed or target image; $\bar{f}$ and $\bar{g}$ are the average gray values for images and, finally, $u$ and $v$ are, respectively, the displacement components for the subwindow centers in the $x$ and $y$ directions.

The CCD camera uses a small rectangular piece of silicon, which has been segmented into an $\mathrm{H} \times \mathrm{V}$ array of individual light sensitive cells, also known as pixel. Each pixel stores a certain gray scale value ranging from 0 to 255 , in accordance with the intensity of the light reflected by the surface of specimen. Concerning the specimen preparation, the aim is to create a random speckle pattern on the specimen surface. Each speckle has a unique shape and intensity and serves as an ideal target. The smaller the grains are, the higher the spatial resolution. The measured results will depend on the CCD pixel resolution, the speckle size, and the DIC software considered.

\section{Parameter Estimation}

In order to fully characterize a mechanical system it is required to estimate a set of unknown parameters which are representative to its dynamics. Therefore, for the sake of simplicity, the general vector $\mathbf{p}$, defined by Eq. (3), contains the information concerning all the unknown parameters for the system under investigation, i.e.,

$$
\mathbf{p}=\left\{p_{1}, p_{2}, \ldots, p_{N_{p}}\right\}^{T}
$$

where $N_{p}$ corresponds to the number of unknown parameters. In the inverse problem formulation, one considers that the set of parameters $\mathbf{p}$ is unknown and that there is available a set of experimental data concerning the response of the system, denoted by the symbol $\mathbf{y}^{E}(\mathbf{x}, t)$, to a certain excitation/stimulus. The basic idea is to find out the set of parameters $\mathbf{p}$ that best correlates the response $\mathbf{y}(\mathbf{p} ; \mathbf{x}, t)$, which is obtained from an appropriate mathematical model for the system under study, with the experimental response $\mathbf{y}^{E}(\mathbf{x}, t)$. Hence, the idea is to define an objective function $S$ in order to quantify the difference between the two responses $\mathbf{y}^{E}(\mathbf{x}, t)$ and $\mathbf{y}(\mathbf{p} ; \mathbf{x}, t)$. Now, if one assumes the hypothesis that the measurement errors have zero mean, constant variance, Gaussian distribution, and that they are additive and noncorrelated, the error function $S$ that provides the minimum variance estimates is the ordinary least squares norm defined as follows (Özişik and Orlande, 2000; Beck and Arnold, 1977):

$$
S(p)=[\overline{\mathbf{Y}}-\mathbf{Y}]^{T}[\overline{\mathbf{Y}}-\mathbf{Y}]
$$

where $\overline{\mathbf{Y}}$ and $\mathbf{Y}$ are, respectively, the vectors containing the measured and estimated responses of the system, and are defined as follows:

$$
\begin{aligned}
& \overline{\mathbf{Y}}^{T}=\left\{\overline{\mathbf{Y}}_{1}^{T}, \overline{\mathbf{Y}}_{2}^{T}, \ldots, \overline{\mathbf{Y}}_{N_{t}}^{T}\right\} \\
& \mathbf{Y}^{T}=\left\{\mathbf{Y}_{1}^{T}, \mathbf{Y}_{2}^{T}, \ldots, \mathbf{Y}_{N_{t}}^{T}\right\}
\end{aligned}
$$

The symbol $(\bullet)^{T}$ appearing on Eqs. (4)-(6) indicates the transpose of $(\bullet)$. The symbol $N_{t}$ appearing in Eqs. (5) and (6) corresponds to the total number of measured data. The column vectors $\overline{\mathbf{Y}}_{j}$ and $\mathbf{Y}_{j}$ contain, respectively, the experimental and estimated information. 
They are organized such that their $s$-th components $\left[\overline{\mathbf{Y}}_{j}\right]_{s}$ and $\left\lfloor\mathbf{Y}_{j}\right\rfloor_{s}$ represent, respectively, the $j$-th measurement and the $j$-th estimate corresponding to the $s$-th sensor $s=1,2, \ldots, N_{s}\left(N_{s}\right.$ denotes the total number of sensors). It should be emphasized that $\overline{\mathbf{Y}}_{j}$ and $\mathbf{Y}_{j}$ can be displacements, accelerations, strains, natural frequencies, just to cite a few examples.

The inverse problem consists in determining the set of parameters $\mathbf{p}$ which minimizes the objective function, viz.,

$$
\min _{\mathbf{p} \in P} S(\mathbf{p})
$$

where every constraint associated to the inverse problem is represented by the solution set $P$. The inverse problem defined by Eq. (7) will be solved, in the present article, through the LevenbergMarquardt parameter estimation method, which is a well-known and powerful iterative method for solving nonlinear least squares problems of parameter estimation (Özişik and Orlande, 2000; Beck and Arnold, 1977). The iterative procedure of the LevenbergMarquardt method is given by

$$
\mathbf{p}^{k+1}=\mathbf{p}^{k}+\left[\left(\mathbf{J}^{k}\right)^{T} \mathbf{J}^{k}+\mu^{k} \boldsymbol{\Gamma}^{k}\right]^{-1}\left(\mathbf{J}^{k}\right)^{T}\left[\overline{\mathbf{Y}}-\mathbf{Y}\left(\mathbf{p}^{k}\right)\right]
$$

where $\mu$ is a stabilization parameter; $\Gamma$ is a diagonal matrix; the superscript $k$ denotes the iteration number; and $\mathbf{J}$ is the sensitivity matrix, defined as follows:

$$
J_{i j}=\frac{\partial Y_{i}}{\partial p_{j}}, i=1,2, \ldots, N_{s} \times N_{t} \text { and } j=1,2, \ldots, N_{p}
$$

The purpose of the term $\mu^{k} \Gamma^{k}$ in Eq. (8) is to reduce the oscillations or instabilities due to the ill-conditioning associated to the inverse problem. The decrease of these instabilities or oscillations can be achieved by adopting a matrix $\mu^{k} \Gamma^{k}$ of which components are relatively large as compared to the components of the matrix $\left(\mathbf{J}^{k}\right)^{T} \mathbf{J}^{k}$ (Özişik and Orlande, 2000). The parameter $\mu^{k}$ is chosen such that $S\left(\mathbf{p}^{k+1}\right)<S\left(\mathbf{p}^{k}\right)$ remains valid at every iteration. The stopping criteria adopted for the iteration process are the ones suggested by Dennis and Schnabel (Özişik and Orlande, 2000), as follows:

$$
\begin{aligned}
& S\left(\mathbf{p}^{k+1}\right)<\varepsilon_{1} \\
& \| \mathbf{J}\left(\mathbf{p}^{k+1}\right)^{T}\left[\overline{\mathbf{Y}}-\mathbf{Y}\left(\mathbf{p}^{k+1}\right)\right] \mid<\varepsilon_{2} \\
& \left\|\mathbf{p}^{k+1}-\mathbf{p}^{k}\right\|<\varepsilon_{3}
\end{aligned}
$$

where $\varepsilon_{1}, \varepsilon_{2}$ and $\varepsilon_{3}$ are user-prescribed tolerances and $\|\bullet\|$ corresponds to the Euclidean norm. Different versions of the Levenberg-Marquardt iterative procedure can be found in the literature, depending on the choice for the diagonal matrix $\Gamma$ and on the form chosen for the variation of the parameter $\mu$ (Özişik and Orlande, 2000). For the present work it has been chosen the matrix $\Gamma$, as follows:

$$
\Gamma^{k}=\operatorname{diag}\left[\left(\mathbf{J}^{k}\right)^{T} \mathbf{J}^{k}\right]
$$

\section{Experimental Set-Up}

The experimental arrangement for the measurement of the fullfield displacement through the DIC method involves a fixed-free cantilever beam, a loading device, a CCD camera set perpendicularly to the specimen and a computer, as shown in Fig. 2. The cantilever beam was covered with painted speckles (random black and white pattern). The CCD camera (Sony XCD-SX910) used to record the speckle image of the specimen before and after the load application has a resolution of $1376 \times 1024$ pixels. In this experimental configuration one pixel of the CCD camera corresponds to an area approximately equal to $4.65 \times 4.65 \mu \mathrm{m}^{2}$ on the specimen.

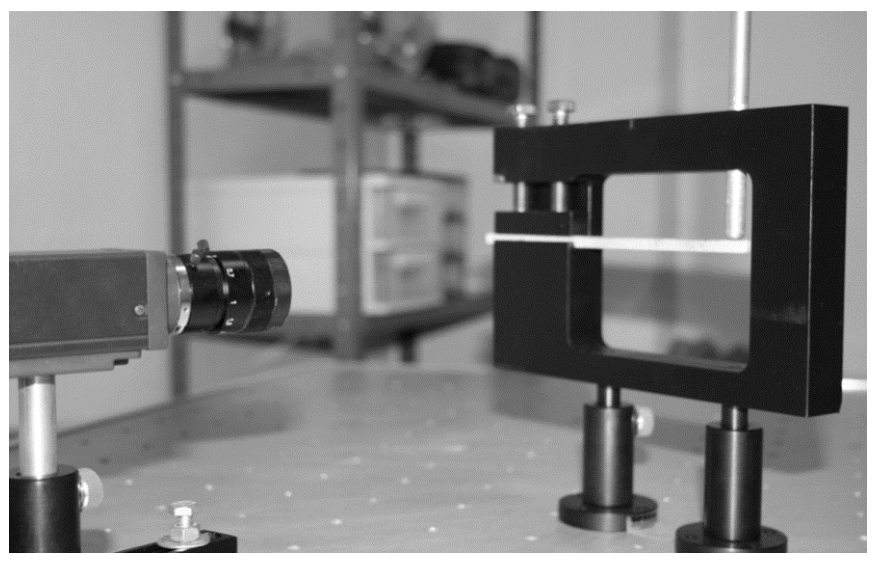

Figure 2. Experimental arrangement of DIC method.

Figure 3 depicts a simple sketch of the experimental set-up illustrated in Fig. 2. A cantilevered beam-like specimen is subjected to a concentrated load $F$ applied at the free end, as shown in Fig. 3.

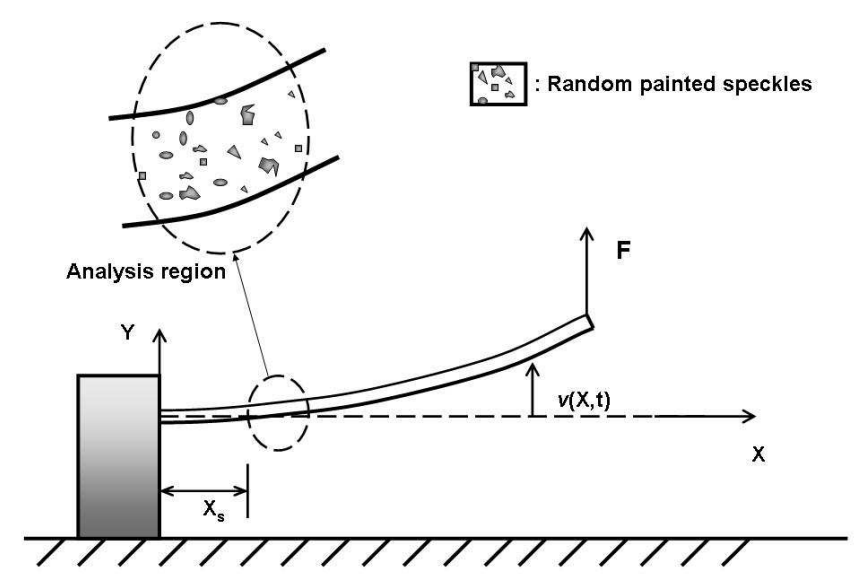

Figure 3. Sketch of the experimental set-up. 
The experimental set-up shown in Fig. 2 is used to measure the displacement field of a specific region of the beam, henceforth referred to as the analysis region. The symbol $x_{s}$, in Fig. 3, denotes the $x$-coordinate of the starting point of the analysis region. Such measured data are used as input data to the Levenberg-Marquardt iterative procedure in order to estimate the beam elasticity modulus.

The transverse displacement field $v(x, t)$ for the elastic cantilever beam shown in Fig. 3 is governed by the following partial differential equation and boundary-conditions:

$$
\begin{aligned}
& \rho \frac{\partial^{2} v(x, t)}{\partial t^{2}}+\frac{\partial^{2}}{\partial x^{2}}\left[E I \frac{\partial^{2} v(x, t)}{\partial x^{2}}\right]=0,0<x<L, t>0 \\
& v(0, t)=0 \text { and } \frac{\partial v}{\partial x}(0, t)=0 \text { at } x=0, t>0 \\
& E I \frac{\partial^{2} v}{\partial x^{2}}(L, t)=0 \text { and }-\frac{\partial}{\partial x}\left[E I \frac{\partial^{2} v}{\partial x^{2}}\right](L, t)=F \text { at } x=L, t>0
\end{aligned}
$$

where $\rho, E$ and $I$ denote, respectively, the specific mass, the elasticity modulus and the beam cross-section moment of inertia. The displacement field measured by the DIC method is used to estimate the elasticity modulus $E$, assumed here as an unknown parameter in Eq. (14). For the experimental set-up shown in Figures (2) and (3) it will be considered only static forces; hence, the inertia term appearing on the left-hand side of the differential equation (14) is disregarded in the present analysis.

\section{Results}

In this section some results concerning the parameter estimation as well as model validation are presented. Two beam-like specimens were extracted from a unique aluminum beam. The first one was used for parameter estimation and the second one was used for model validation.

\section{Estimation}

For the estimation processes, a rectangular cross-section beam was manufactured. Its dimensions, cross-section moment of inertia and magnitude of the applied force are indicated in Table 1. Two images of the beam in the same analysis region, associated with different loads of $0 \mathrm{~N}$ and $10.15 \mathrm{~N}$, are used in order to compute the full-field displacement by means of the DIC method. A DIC-based algorithm has been developed at "Laboratório de Óptica Não Linear \& Aplicada da Universidade Federal Fluminense". Figure 4 illustrates the texture pattern of the underformed (a) and deformed (b) coating specimen surface.

Figure 5 shows the measured full-field displacement $v(x, y)$ along the $x$-axis, when the cantilever beam is subjected to the applied load $F=10.15 \mathrm{~N}$.

Table 1. Dimensions, cross-section moment of inertia and magnitude of the applied force for the cantilever beam shown in Fig. 2.

\begin{tabular}{cccc}
\hline Material: Aluminum & Symbol & Units & Value \\
\hline Moment of inertia & $I$ & $\mathrm{~m}^{4}$ & $6.23 \times 10^{-11}$ \\
Length & $L$ & $\mathrm{~m}$ & 0.101 \\
Height & $h$ & $\mathrm{~m}$ & $5.4 \times 10^{-3}$ \\
Width & $b$ & $\mathrm{~m}$ & $4.75 \times 10^{-3}$ \\
Applied force & $F$ & $\mathrm{~N}$ & 10.15 \\
\hline
\end{tabular}

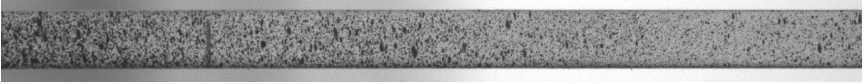

(a)

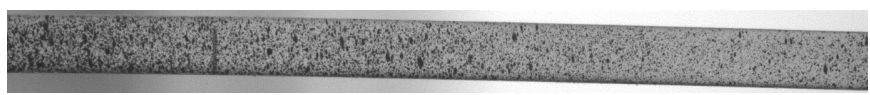

(b)

Figure 4. Pattern of the coating specimen: (a) undeformed $(F=0 \mathrm{~N})$, and (b) deformed $(F=10.15 \mathrm{~N})$.

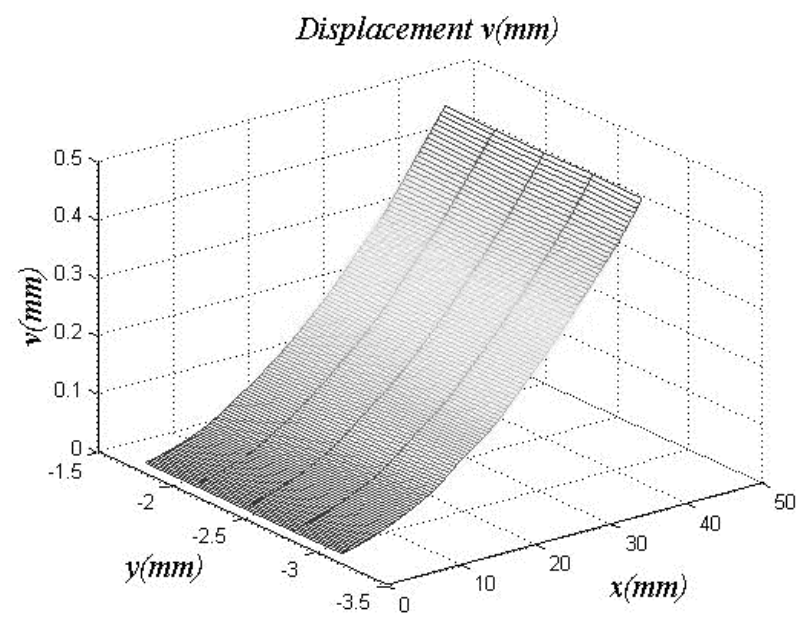

Figure 5. Transverse displacement field, $v(x, y)$, for the applied load $\boldsymbol{F}=$ $10.15 \mathrm{~N}$.

During the estimation processes, the boundary-value problem given by Eq. (14) (also known as the direct or forward problem in the inverse problem terminology) is solved through the finite element method (Reddy, 2006). The error function $S$ given by Eq. (4) comprises the difference between the measured displacement field and the one derived from the finite element solution. Taking for granted that the geometry of the specimen and the applied load are measured accurately, the primary interest is to estimate the elasticity modulus $E$ appearing in Eq. (14). Nevertheless, after some data acquisition, one can conclude that there is some level of uncertainty concerning the coordinate $x_{s}$ of the starting point of the analysis region. Hence, in order to overcome the aforementioned shortcoming, the vector of unknown parameters $\mathbf{p}$ contains two components: (i) the elasticity modulus $E$, parameterized as $E=p_{1} \times 10^{9}(\mathrm{~Pa})$, and (ii) the coordinate $x_{s}$ parameterized as $x_{s}=p_{2}(\mathrm{~m})$.

The addition of a second parameter to be estimated naturally raises a question about the feasibility of estimating $p_{1}$ and $p_{2}$ at the same time. Hence, it is required some sensitivity analyses. Local sensitivity analyses for different reference parameter vectors are shown in Fig. 6 . This figure depicts the ratio between the sensitivity coefficients $\partial v / \partial p_{1}$ and $\partial v / \partial p_{2}$. According to Fig. 6, for the chosen sets of parameter vectors, one may conclude that there is no linear dependence between these sensitivity coefficients; therefore, the two parameters may be estimated simultaneously. 


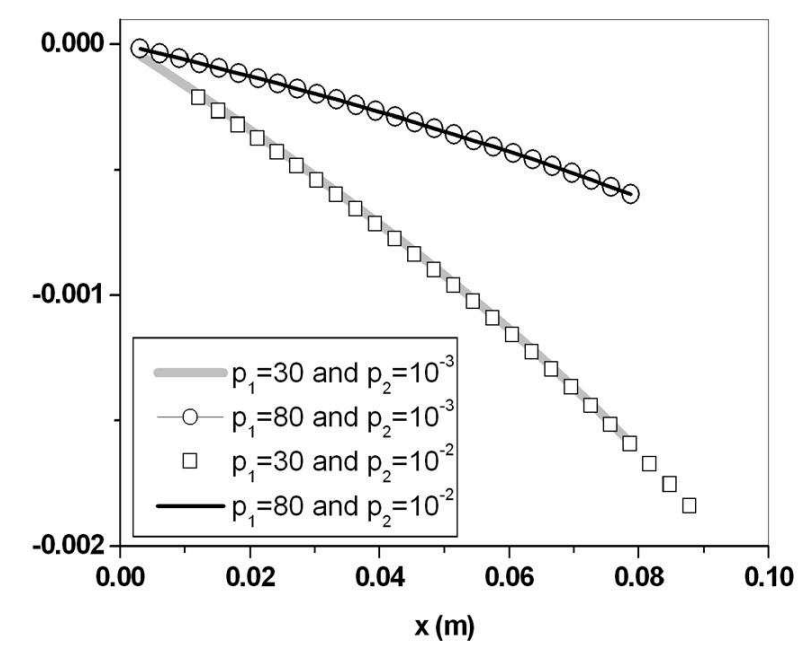

Figure 6. Ratio of the sensitivity coefficients $\partial v / \partial p_{1}$ and $\partial v / \partial p_{2}$ for different reference parameters.

The estimation process considered four different initial guesses: (i) $\mathbf{p}^{(0)}=\left\{30,10^{-3}\right\}^{\mathrm{T}}$, (ii) $\mathbf{p}^{(0)}=\left\{80,10^{-3}\right\}^{\mathrm{T}}$, (iii) $\mathbf{p}^{(0)}=\left\{30,10^{-2}\right\}^{\mathrm{T}}$, and (iv) $\mathbf{p}^{(0)}=\left\{80,10^{-2}\right\}^{\mathrm{T}}$. It was also considered two different finite element meshes, with 50 and 100 finite elements for the solution of the associated direct problem. All of the estimations provided the same vector of estimated parameters $\hat{\mathbf{p}}=\left\{67.70,1.91 \times 10^{-2}\right\}^{T}$.

Figure 7 depicts the residue $R(\hat{\mathbf{p}})$ along the $x$-coordinate. The residue $R(\hat{\mathbf{p}})$ is defined as the difference between the measured displacement $v^{E}(x)$ and the one computed from the finite element model $v(\hat{\mathbf{p}} ; x)$. The residue shown in Fig. 7 has a random signature, which corroborates the adequacy of the mathematical model (Beck and Arnold, 1977). Nevertheless, in order to definitely validate the estimation procedure and the associated mathematical model, it is necessary to consider an experimental data set different from the one used for the estimation processes (Roache, 1998).

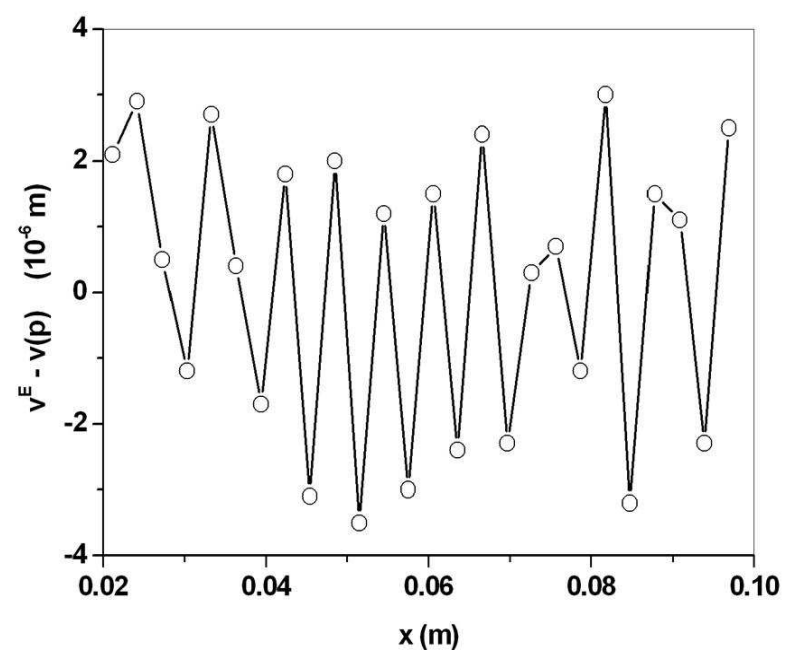

Figure 7. Residue $R(\hat{\mathbf{p}} ; \mathbf{x})=v^{E}(\mathbf{x})-v(\hat{\mathbf{p}} ; \mathbf{x})$ along the $\boldsymbol{x}$-coordinate.

\section{Validation}

In order to validate the estimated parameter and the associated mathematical model described in the previous section, it was considered a different set of experimental data out of a different experimental set-up. It was prepared another beam-like specimen, which is made of the same material as the one analyzed in the previous section. This new specimen possesses different dimensions from the first specimen, as shown in Fig. 8, and it was subjected to a classical modal analysis test. The measured data obtained from this new experiment were used for validating the parameter estimated in previous section. They were also used to estimate the elasticity modulus of the beam-like specimen shown in Fig. 8. The estimation performed in this section was also done by the LevenbergMarquardt technique.

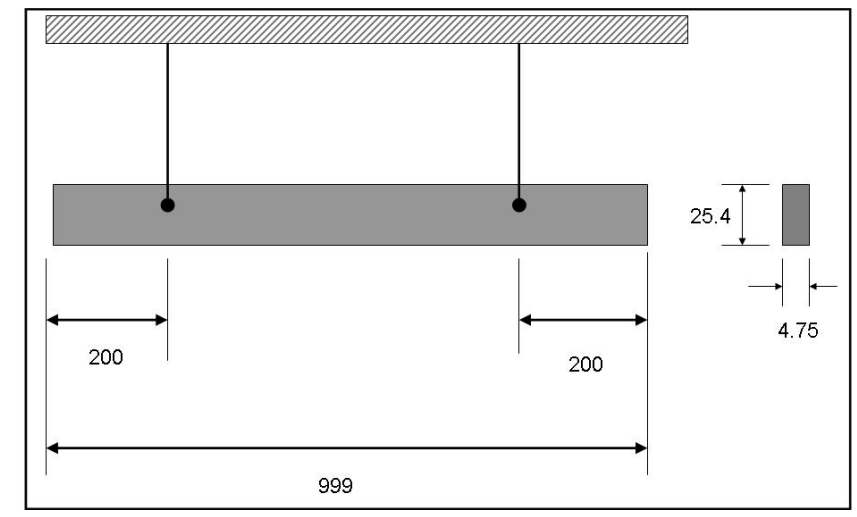

Figure 8. Sketch of the free-free beam (dimensions in $\mathrm{mm}$ ) used for modal analysis tests.

A sketch of the experimental set-up for the second beam-like specimen is shown in Fig. 8. The beam was suspended by lightweight elastic cords and it was instrumented with a piezoelectric accelerometer located at one of its ends. The model of the accelerometer is PCB 353M197 and its mass is equal to $9.71 \mathrm{~g}$. The beam was excited with an impact hammer and its first five natural frequencies were measured. The measured natural frequencies are: $f_{l}=23.25 \mathrm{~Hz} ; f_{2}=64.65 \mathrm{~Hz} ; f_{3}=127.5 \mathrm{~Hz}$; $f_{4}=212 \mathrm{~Hz}$; and $f_{5}=317 \mathrm{~Hz}$.

Two error functions were considered for the estimation of the beam-like elasticity modulus shown in Fig. 8 . The estimates for the beam-like specimen shown in Fig. 8 are in good agreement with the one previously obtained. These estimates are presented in Table 2.

Table 2. Estimates obtained for the elasticity modulus.

\begin{tabular}{ccc}
\hline Error Function $S$ & Beam-like specimen & $\hat{E}(\mathrm{Gpa})$ \\
\hline$\sum_{j=1}^{5}\left(f_{j}(\mathbf{p})-f_{j}{ }^{E x p}\right)^{2}$ & Figure 8 & 68.7 \\
$\sum_{j=1}^{5}\left(\frac{f_{j}(\mathbf{p})-f_{j}{ }^{E x p}}{f_{j}^{E x p}}\right)^{2}$ & Figure 8 & 68.3 \\
$\sum_{j=1}^{25}\left(v\left(\mathbf{p} ; x_{j}\right)-v^{E}\left(x_{j}\right)\right)^{2}$ & Figures 2 and 3 & 67.7 \\
\hline
\end{tabular}


Figure 9 summarizes the validation data by depicting the relative error between the measured $f_{j}^{\exp }$, and the estimated $f_{j}$, natural frequencies $j=1,2, \ldots, 5$. The estimated natural frequencies are computed from the finite-element solution of the boundary-value problem given by Eq. (14), using the estimated elasticity modulus indicated in Table 2.

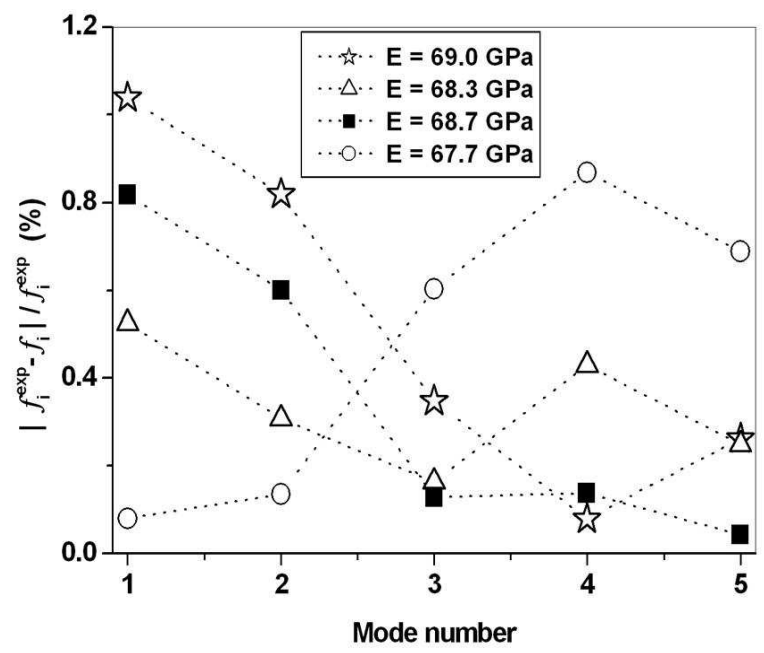

Figure 9. Validation graph.

In Fig. 9, the variation $\Delta f_{j}=f_{j}{ }^{\exp }-f_{j}, j=1,2, \ldots, 5$, was computed for four different values of the beam elasticity modulus $E$. The first one $E=69 \mathrm{GPa}$ is the one commonly found in the reference books for aluminum material; $E=68.3 \mathrm{GPa}$ and $E=68.7$ $\mathrm{GPa}$ are the estimates obtained for the beam-like specimen, shown in Fig. (8), through the minimization of the first two error functions, shown in Table (2); and, finally, $E=67.7 \mathrm{GPa}$ is the estimate obtained for the beam-like specimen, shown in Figs. (2) and (3), through the minimization of the last error function, shown in Table (2). The relative errors in the first five natural frequencies associated to the estimate $E=67.7 \mathrm{GPa}$ are less than $1 \%$, which corroborates the estimation obtained in the estimation subsection. As it has been previously mentioned, this validation procedure considered a different experimental set-up and a different beam-like specimen from the one used in the estimation subsection.

\section{Concluding Remarks}

In the present work, the Digital Image Correlation (DIC) measurement method is used to obtain the displacement field of specific regions of a cantilever beam under bending. These fields are used within an inverse analysis scheme in order to obtain constitutive parameters for beam-like specimens. The parameter estimation is performed by means of the minimization of an error function comprising of the difference between the displacement fields obtained from the experiment and from an appropriate mathematical model. The inverse problem is solved by means of the classical Levenberg-Marquardt nonlinear parameter estimation technique.

As an example, we estimate the elasticity modulus of an aluminum beam-like specimen. The estimated parameter and the associated mathematical model were validated taking into account measured data extracted from an experimental set-up completely different from the one used for the estimation process.

The main contribution of this work is to provide a low-cost alternative path for the estimation of elastic parameters based on digital image methods.

\section{Acknowledgements}

The authors would like to express their gratitude to $\mathrm{Mr}$. Anderson Pessoa de Souza from LAVI - "Laboratório de Acústica e Vibrações" of Federal University of Rio de Janeiro as well as to the Ministry of Science and Technology through its research funding agency $\mathrm{CNPq}$.

\section{References}

Beck, J.V. and Arnold, K.J., 1977, "Parameter Estimation in Engineering and Science", John Wiley \& Sons, New York.

Burke, B.C.P., Kim, S.O, Kim, K.S., 2007, "Partial polar decomposition inverse method applied to determination of internal stresses in an elastic complex structure", International Journal of Solids and Structures, Vol. 44, pp. 2010-2020.

Cardenas-Garcia, J.F., Preidikman, S., 2006, "Solution of the Moiré hole drilling method using a finite-element-method-based approach", International Journal of Solids and Structures, Vol. 43, pp. 6751-6766.

Corr, D., Accardi, M., Graham-Brady, L., Shah, S., 2007, "Digital image correlation analysis of interfacial debonding properties and fracture behavior in concrete", Engineering Fracture Mechanics, Vol.74, pp. 109-121.

Dally, J.W. and Riley, W.F., 2005, "Experimental Stress Analysis", 4 ed., McGraw Hill.

Lecompte, D., Smits, A., Sol, H., Vantomme, J., Hemelrijck, D.V., 2007, "Mixed numerical-experimental technique for orthotropic parameter identification using biaxial tensile tests on cruciform specimens", International Journal of Solids and Structures, Vol. 44, No. 5, pp. 1643-1656.

Leiderman, R., Barbone, P.E., Braga, A.M.B., 2007, "Reconstructing the adhesion stiffness distribution in a laminated elastic plate: Exact and approximate inverse scattering solutions", Journal of the Acoustical Society of America, Vol. 122, pp. 1906-1916.

McGinnis, M.J., Pessiki, S. and Turker, H., 2005, "Application of Three-dimensional Digital Image Correlation to the Core-drilling Method", Experimental Mechanics, Vol. 45, No. 4, pp. 359-366.

Özişik, M.N. and Orlande, H.R.B, 2000, "Inverse Heat Transfer: Fundamentals and Applications", Taylor and Francis.

Pandurangan, P., Buckner, G.D., 2007, "Defect identification in GRIDLOCK® joints", NDT\&E International, Vol. 40, pp. 347-356.

Reddy, J.N., 2006, "An Introduction to the Finite Element Method", McGraw Hill, 3rd Edition.

Roache, P.J., 1998, "Verification and Validation in Computational Science and Engineering", Hermosa Publisher.

Stutz, L.T., Castello, D.A. and Rochinha, F.A., 2005, "A flexibilitybased continuum damage identification approach", Journal of Sound and Vibration, Vol. 279, Issues 3-5, pp. 641-667.

Wang, Y., Cuitino A.M., 2002, "Full-field measurements of heterogeneous deformation patterns on polymeric foams using digital image correlation", International Journal of Solids and Structures, Vol. 39, pp. 3777-3796. 